\title{
Parallel Dialogue Games and Hypersequents for Intermediate Logics
}

\author{
Christian G. Fermüller \\ Technische Universität Wien, Austria
}

\begin{abstract}
A parallel version of Lorenzen's dialogue theoretic foundation for intuitionistic logic is shown to be adequate for a number of important intermediate logics. The soundness and completeness proofs proceed by relating hypersequent derivations to winning strategies for parallel dialogue games. This also provides a computational interpretation of hypersequents.
\end{abstract}

\section{Introduction}

In recent years hypersequent calculi have emerged as a flexible type of proof system for a wide range of logics (see, e.g., [3, 5, 4]). These calculi share many favorable properties with Gentzen's classic sequent calculi LK and LI. Most importantly, cuts are eliminable and the logical rules are strictly analytic - i.e., they only refer to immediate subformulas of the introduced formula and are context independent. Consequently these calculi are of relevance to automated reasoning. However, the relation between hypersequent derivations and the semantics of the corresponding logics is much more obscure than in the case of classical or intuitionistic sequents. Standard completeness proofs for $\mathbf{L K}$ and $\mathbf{L I}$ show how to extract counter models for underivable formulas; it is mainly this feature that allows to call (a particular form of) goal oriented proof search in sequent calculi 'semantic tableaux'. Unfortunately, the hypersequent calculi that have been formulated for intermediate logics like Gödel-Dummett logic $\mathbf{G}_{\infty}$, the logic $\mathbf{L Q}$ of weak excluded middle, or finite-valued Gödel logics do not relate directly to a semantic foundation of these logics. To address this concern, we show that hypersequents bear a close relation to an interesting foundational approach that constitutes an alternative to standard Tarski-style semantics: dialogue games.

\section{Lorenzen style dialogue games}

Logical dialogue games come in many forms and versions, nowadays. Here, we do not use more recent formulations in the style of Blass [2] or Abramsky [1] ${ }^{1}$, but rather refer directly to Paul Lorenzen's original idea (dating back to the late 1950s, see e.g., [15]) to identify logical validity of a formula $A$ with the existence of a winning strategy for

\footnotetext{
${ }^{1}$ These more modern logical dialogue games differ considerably from the orginal ones of Lorenzen and his school. In particular, parallelism is introduced already at the level of analyzing single connectives. This feature of Blass/Abramsky style games makes them useful for modelling certain features of linear logic and related formalisms, but less well connected to the well motivated foundational intentions of Lorenzen.
} 
a proponent $\mathbf{P}$ in an idealized confrontational dialogue, in which $\mathbf{P}$ tries to uphold $A$ against systematic doubts by an opponent $\mathbf{O}$. Although the claim that this leads to an alternative characterization — or even: 'justification' — of intuitionistic logic was implicit already in Lorenzen's early essays, it took more than twenty years until the first rigorous, complete and error free proof of this central claim was published in [8]. Many variants of Lorenzen's original dialogue games have appeared in the literature since. (Already Lorenzen and his collaborators defined different versions of the game. See, eg., $[9,13]$ for further references.) Here, we define a version of dialogue games that are: 1) well suited for demonstrating the close relation to analytic Gentzen-type systems; 2) easily shown to be equivalent to other versions of dialogue games for intuitionistic logic, that can be found in the literature; 3) straightforward to consider 'in parallel'.

Notation. An atomic formula (atom) is either a propositional variable or $\perp$ (falsum). As usual, compound formulas are built up from atoms using the connectives $\supset, \wedge, \vee$; $\neg A$ abbreviates $A \supset \perp$. In addition to formulas, the special signs ?, I?, r? can be stated in a dialogue by the players $\mathbf{P}$ and $\mathbf{O}$, as specified below.

Dialogue games are characterized by two sorts of rules: logical ones and structural ones. The logical rules define how to attack a compound formula and how to defend against such an attack. They are summarized in the following table. (If $\mathbf{X}$ is the proponent $\mathbf{P}$ then $\mathbf{Y}$ refers to the opponent $\mathbf{O}$, and vice versa. ${ }^{2}$ )

\section{Logical dialogue rules:}

\begin{tabular}{|c|c|c|}
\hline $\mathbf{X}:$ & attack by $\mathbf{Y}$ & defense by $\mathbf{X}$ \\
\hline \hline$A \wedge B$ & I? or $\mathrm{r}$ ? (Y chooses) & $A$ or $B$, accordingly \\
\hline$A \vee B$ & $?$ & $A$ or $B$ (X chooses) \\
\hline$A \supset B$ & A & B \\
\hline
\end{tabular}

We will see below that $\mathbf{O}$ may also attack atoms (including $\perp$ ) by stating '?'.

A dialogue is a sequence of moves, which are either attacking or defending statements, in accordance with the logical rules. Each dialogue refers to a finite multiset of formulas that are initially granted by $\mathbf{O}$, and to an initial formula to be defended by $\mathbf{P}$.

Moves can be viewed as state transitions. In any state of the dialogue the (multiset of) formulas, that have been either initially granted or stated by $\mathbf{O}$ so far, are called the granted formulas (at this state). The last formula that has been stated by $\mathbf{P}$ and that either already has been attacked or must be attacked in O's next move is called active formula. (Note that the active formula, in general, is not the last formula stated by $\mathbf{P}$; since $\mathbf{P}$ may have stated formulas after the active formula, that are not attacked by $\mathbf{O}$.) With each state of a dialogue we thus associate a dialogue sequent $\Pi \vdash A$, where $\Pi$ denotes the granted formulas and $A$ the active formula.

We stipulate that each move carries the information (pointers) necessary to reconstruct which formula is attacked or defended in which way in that move. However, we do not care about the exact way in which this information is coded.

Structural rules (Rahmenregeln in the diction of Lorenzen and his school) regulate the succession of moves. Quite a number of different systems of structural rules have been proposed in the literature (See e.g., [16,9,13]; in particular, [13] compares and

\footnotetext{
${ }^{2}$ Note that both players may launch attacks as well as defending moves during the course of a dialogue. For motivation and detailed exposition of these rules we refer to [9].
} 
discusses different systems.). The following rules, together with the winning conditions stated below, amount to a version of dialogues traditionally called $E i$-dialogues (i.e., Felscher's E-dialogues combined with the so-called ipse dixisti rule; see, e.g., [13]).

\section{Structural dialogue rules:}

Start: The first move of the dialogue is carried out by $\mathbf{O}$ and consists in an attack on the initial formula.

Alternate: Moves strictly alternate between players $\mathbf{O}$ and $\mathbf{P}$.

Atom: Atomic formulas, including $\perp$, may be stated by both players, but can neither be attacked nor defended by $\mathbf{P}$.

$E$ : Each (but the first) move of $\mathbf{O}$ reacts directly to the immediately preceding move by $\mathbf{P}$. I.e., if $\mathbf{P}$ attacks a granted formula then $\mathbf{O}$ 's next move either defends this formula or attacks the formula used by $\mathbf{P}$ to launch this attack. If, on the other hand, P's last move was a defending one then $\mathbf{O}$ has to attack immediately the formula stated by $\mathbf{P}$ in that defense move.

\section{Winning conditions (for $\mathbf{P}$ ):}

$W$ : The game ends with $\mathbf{P}$ winning if $\mathbf{O}$ has attacked a formula that has already been granted (either initially or in a later move) by $\mathbf{O}$.

$W \perp$ : The game ends with $\mathbf{P}$ winning if $\mathbf{O}$ has granted $\perp$.

A dialogue tree $\tau$ for $\Pi \vdash C$ is a rooted directed tree with nodes labelled by dialogue sequents and edges corresponding to moves, such that each branch of $\tau$ is a dialogue with initially granted formulas $\Pi$ and initial formula $C$. We thus identify the nodes of a dialogue tree with states of a dialogue. We distinguish $\mathbf{P}$-nodes and $\mathbf{O}$-nodes, according to whether it is P's or O's turn to move at the corresponding state.

A finite dialogue tree is a winning strategy (for $\mathbf{P}$ ) if the following conditions hold:

1. Every $\mathbf{P}$-node has at most one successor node.

2. All leaf nodes are $\mathbf{P}$-nodes in which the winning conditions for $\mathbf{P}$ are fulfilled.

3. Every $\mathbf{O}$-node has a successor node for each move by $\mathbf{O}$ that is a permissible continuation of the dialogue (according to the rules) at this stage.

Winning strategies for a player in a non-cooperative two-person game are more commonly described as functions assigning a move for that player to each state of the game, taking into account all possible moves of the opponent. Observe that our tree form of a winning strategy just describes the corresponding function in a manner that makes the step-wise evolution of permissible dialogues more explicit.

As already mentioned, a dialogue game may be viewed as a state transition system, where moves in a dialogue correspond to transitions between $\mathbf{P}$-nodes and $\mathbf{O}$-nodes. A dialogue then is a possible trace in the system; and a winning strategy can be obtained by a systematic 'unraveling' of all possible traces.

To illustrate this point, consider the implicational fragment of the language; i.e., the set of formulas not containing $\wedge$ or $\vee$. Henceforth we use the following notation: For every compound formula $F$ of form $C \supset D, F_{p}$ denotes $C$ and $F_{c}$ denotes $D$. If $F$ is atomic then $F_{p}$ is empty (and $F_{c}$ remains undefined). $F_{p p}$ is $C_{p}$ if $F=C \supset D$. The figure, below, represents all permissible moves in a dialogue for this fragment. By labelling a transition with $\Pi \stackrel{+}{\longleftarrow} F$ we denote that $F$ is added to the multiset $\Pi$ of granted formulas. $A \leftarrow C$ means that $C$, as a result of the corresponding move, is the new active formula. 
Dialogue as state transitions $(\supset)$ :

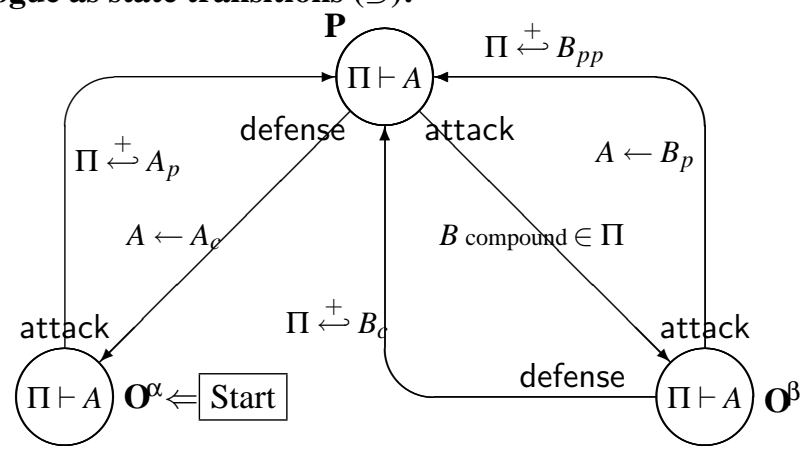

The encircled labels denote the dialogue sequent at the corresponding state. The edges from the $\mathbf{P}$-node to the two $\mathbf{O}$-nodes correspond to the principal choice of player $\mathbf{P}$ : either to defend the active formula or to attack a compound formula $B$ from the granted formulas. (The fact that $A_{c}$ is undefined if $A$ is atomic means that in this case the transition from node $\mathbf{P}$ to node $\mathbf{O}^{\alpha}$ is not possible. This corresponds to the stipulation of rule Atom, that atomic formulas cannot be defended by $\mathbf{P}$. However, remember that the dialogue is already in a winning state for $\mathbf{P}$ if the active formula $A$ is among the granted formulas $\Pi$.)

On the other hand, according to the structural rule $E$, player $\mathbf{O}$ has no choice but to attack the last formula of $\mathbf{P}$ if $\mathbf{P}$ 's last move was a defense (i.e., if the dialogue is in state $\mathbf{O}^{\alpha}$ ). In state $\boldsymbol{O}^{\beta}$, however, $\mathbf{O}$ may either defend the attacked formula or attack the formula used by $\mathbf{P}$ in launching the last attack ('counter-attack').

The winning conditions have to be checked at state $\mathbf{P}$ only. If $\perp \in \Pi$ or $A \in \Pi$ then the game ends in that state with $\mathbf{P}$ winning.

Adding $\wedge$ and $\vee$ to the language amounts to adding further possible transitions (between the nodes $\mathbf{P}$ and $\mathbf{O}^{\alpha}$, and $\mathbf{P}$ and $\mathbf{O}^{\beta}$, respectively).

\section{Basic adequateness of dialogues}

Proving the adequateness of dialogue games for intuitionistic logic consists in showing that winning strategies can be transformed into (analytic) proofs of Gentzen's well known sequent calculus $\mathbf{L I}$ for intuitionistic logic, and vice versa. ${ }^{3}$

To this aim, we use the following variant $\mathbf{L I}^{\prime}$ of $\mathbf{L I}$ :

Axioms: $\perp, \Pi \longrightarrow C$ and $A, \Pi \longrightarrow A$

Logical rules: ${ }^{4}$

$$
\begin{array}{ll}
\stackrel{A, A \vee B, \Pi \longrightarrow C}{\longrightarrow} \quad B, A \vee B, \Pi \longrightarrow C & (\vee, l) \quad \frac{\Pi \longrightarrow A_{i}}{\Pi \longrightarrow A_{1} \vee A_{2}}\left(\vee_{i}, r\right) \\
\frac{A_{i}, A_{1} \wedge A_{2}, \Pi \longrightarrow C}{A_{1} \wedge A_{2}, \Pi \longrightarrow C}\left(\wedge_{i}, l\right) & \frac{\Pi \longrightarrow A}{\Pi \longrightarrow C}(\wedge, r)
\end{array}
$$

\footnotetext{
${ }^{3}$ Quite a few proofs of the adequateness of dialogue games for characterizing intuitionistic logic can be found in the literature. Since we will build directly on such a proof - also in going beyond intuitionistic logic - we have to present our own version of it, which draws on ideas from $[13,14]$ and $[8]$ but differs in a number of essential details.

${ }^{4}$ Since $\perp$ is in the language, we do not have to consider empty right hand sides of sequents.
} 


$$
\stackrel{A \supset B, \Pi \longrightarrow A \quad B, A \supset B, \Pi \longrightarrow C}{\longrightarrow}(\supset, l) \quad \frac{A, \Pi \longrightarrow B}{\Pi \longrightarrow A \supset B}(\supset, r)
$$

Structural rules: These are the usual weakening, contraction and cut rules.

It is straightforward to check that $\mathbf{L} \mathbf{I}^{\prime}$ is sound and complete for intuitionistic logic. As a corollary, the following holds:

Proposition 1. $A, \Gamma \longrightarrow A \supset B$ is provable in $\mathbf{L I}^{\prime}$ only if $\Gamma \longrightarrow A \supset B$ is provable.

Theorem 1. Every winning strategy $\tau$ for $\Gamma \vdash C$ (i.e., for dialogues with initial formula $C$, where player $\mathbf{O}$ initially grants the formulas in $\Gamma)$ can be transformed into an $\mathbf{L} \mathbf{I}^{\prime}$ proof of $\Gamma \longrightarrow C$.

Proof. We prove by induction on the depth $d$ of $\tau$ that for every $\mathbf{P}$-node of $\tau$ there is an $\mathbf{L I}$-proof of the sequent corresponding to the dialogue sequent at this node. That this implies the theorem is obvious for the cases where $C$ is either atomic, or a disjunction, or a conjunction; because, in those cases, the dialogue sequent at the $\mathbf{P}$-node(s) immediately succeeding the root node is (are) identical to $\Gamma \vdash C$. In the case where $C=A \supset B$, the $\mathbf{P}$-node succeeding the root carries $A, \Gamma \vdash A \supset B$ as dialogue sequent; and thus the theorem follows from Proposition 1.

The base case, $d=1$, follows from the fact that the $\mathbf{P}$-node (or, in case of $C$ being a conjunction, the two $\mathbf{P}$-nodes) succeeding the root is a (are) leaf node(s). This implies that one of the winning conditions $-C \in \Gamma$ or $\perp \in \Gamma$ - must hold. Consequently, the corresponding sequent $\Gamma \longrightarrow C$ is an axiom.

For $d>1$ we have to distinguish cases according to the form of the active formula that is defended or the (compound) formula that is attacked by $\mathbf{P}$. To keep the proof concise, we will only elaborate it for the implicational fragment of the language; it is straightforward to augment the proof to cover also conjunctions and disjunctions.

1. P defends $A \supset B$ : Let $A, \Pi \vdash A \supset B$ be the dialogue sequent at the current $\mathbf{P}$-node. $\mathbf{P}$ moves from the $\mathbf{P}$-node to the $\mathbf{O}^{\alpha}$-node by stating $B$. $\mathbf{O}$ has to reply with a move attacking $B$. We distinguish two cases:

(a) If $B$ is an atom then the attack consists in stating '?'. Thus we return to a $\mathbf{P}$ node with dialogue sequent $A, \Pi \vdash B$. By the induction hypothesis there is an $\mathbf{L I}^{\prime}$-proof of $A, \Pi \longrightarrow B$, which can be extended to a proof of $A, \Pi \longrightarrow A \supset B$ by applying rule $(\supset, r)$ and weakening.

(b) If $B$ is of form $B_{p} \supset B_{c}$ then $\mathbf{O}$ has to attack $B$ by adding $B_{p}$ to the granted formulas $\Pi$. Thus we return to a $\mathbf{P}$-node with dialogue sequent $A, B_{p}, \Pi \vdash B$. By the induction hypothesis there is an $\mathbf{L I}^{\prime}$-proof of $A, B_{p}, \Pi \longrightarrow B$. By Proposition 1 we obtain an $\mathbf{L} \mathbf{I}^{\prime}$-proof of $A, \Pi \longrightarrow B$. The required proof of $A, \Pi \longrightarrow A \supset B$ is obtained by applying rule $(\supset, r)$ and weakening.

2. P attacks $D \supset E$ : Let $D \supset E, \Pi \vdash A$ be the dialogue sequent at the current $\mathbf{P}$-node. $\mathbf{P}$ 's attack consists in stating $D$. (The move refers to the edge from node $\mathbf{P}$ to node $\mathbf{O}^{\beta}$ in the state transition diagram, above.) The strategy then branches since $\mathbf{O}$ may either defend the implication or attack $D$.

(a) If $\mathbf{O}$ chooses to attack $D$ then $D_{p}$ is added to the granted formulas if $D=D_{p} \supset$ $D_{c}$. If $D$ is atomic the multiset of granted formulas remains unchanged. In any case, $D$ is the new active formula at the succeeding $\mathbf{P}$-node. The corresponding dialogue sequent is (1) $D_{p}, D \supset E, \Pi \vdash D$, where $D_{p}$ is empty if $D$ is atomic. 
(b) If, on the other hand, $\mathbf{O}$ chooses to defend $D \supset E$ then it has to grant $E$. The active formula at the succeeding $\mathbf{P}$-node remains $A$. The corresponding dialogue sequent is (2) $E, D \supset E, \Pi \vdash A$.

By the induction hypothesis there are $\mathbf{L} \mathbf{I}^{\prime}$-proofs of the sequents corresponding to (1) and (2). By Proposition 1 we may remove $D_{p}$ from the left hand side of the sequent corresponding to (1). Therefore we obtain a proof of $D \supset E, \Pi \longrightarrow A$ by combining the two proofs with an application of rule $(\supset, l)$.

Remark 1. For proving the soundness of dialogue games (by this we mean that winning strategies only exist for intuitionistically valid sequents) it would in fact not have been necessary to refer to formal derivations. It rather suffices to check that intuitionistic validity transfers from the leaves of a dialogue tree upwards to the root. However for the following completeness proof the special format of the intuitionistic proofs is essential.

The 'weakening friendly' formulation of the axioms and rules of $\mathbf{L I}$ ' allows to eliminate applications of the weakening rule. (Weakenings in $\mathbf{L I}^{\prime}$-proofs can be moved upwards to the axioms, where they are obviously redundant.) Also the contraction rule becomes redundant if we disregard multiple occurrences of the same formula in the left hand side of a sequent. Most importantly, $\mathbf{L I}^{\prime}$ is complete also without cut. Let as refer to a proof that does not contain any applications of structural rules as strongly analytic. The following proposition then sums up the just made observations.

Proposition 2. There is a strongly analytic proof in $\mathbf{L I}^{\prime}$ for $\Gamma \longrightarrow C$ if and only if $\Gamma^{\prime} \longrightarrow$ $C$ is provable in $\mathbf{L I}^{\prime}$, where $\Gamma^{\prime}$ equals $\Gamma$ if taken as set (i.e., if multiple memberships of the same element are discarded).

Theorem 2. Every strongly analytic $\mathbf{L I}^{\prime}$-proof $\pi$ of $\Gamma \longrightarrow C$ can be transformed into a winning strategy for $\Gamma \vdash C$.

Proof. We proceed by induction on the depth of $\pi$. Again, we show the theorem only for the implicational fragment of the language.

If $\Gamma \longrightarrow C$ is an axiom the winning strategy (consisting of two nodes) is obvious. There are two cases to consider for the induction step.

1. $\pi$ ends with an application of $(\supset, r)$ : The end sequent is of form $\Gamma \longrightarrow A \supset B$. By the induction hypothesis there is a winning strategy $\tau$ for $A, \Gamma \vdash B$. $\tau$ can be extended to a winning strategy for $\Gamma \vdash A \supset B$ as follows. We define a new root node; i.e., an $\mathbf{O}^{\alpha}$-node with dialogue sequent $\Gamma \vdash A \supset B$. To this root we attach an edge that leads to a new $\mathbf{P}$-node. The corresponding move of $\mathbf{O}$ consists in granting $A$ as an attack on $A \supset B$. Therefore the dialogue sequent at the new $\mathbf{P}$-node is $A, \Gamma \vdash A \supset B$. We now only have to add an edge from this node to the root node of $\tau$. This edge corresponds to $\mathbf{P}$ stating $B$ in defense of $A \supset B$.

2. $\pi$ ends with $(\supset, l)$ : The end sequent is of form $A \supset B, \Gamma \longrightarrow C$.

By the induction hypothesis there is a winning strategy $\tau_{1}$ for $A \supset B, \Gamma \vdash A$, and a winning strategy $\tau_{2}$ for $B, A \supset B, \Gamma \vdash C$. Let $\tau_{1}^{-}$be the tree, rooted in a $\mathbf{P}$-node with dialogue sequent $A \supset B, C_{p}, A_{p}, \Gamma \vdash A$, that is obtained from $\tau_{1}$ by removing its root 
and adding $C_{p}$ to the granted formulas. We appeal to the general fact that a winning strategy for $\Pi \vdash F$ is also a winning strategy for $C, \Pi \vdash F$. Similarly let $\tau_{2}^{-}$be the tree obtained form $\tau_{2}$ that is rooted in a $\mathbf{P}$-node with dialogue sequent $B, C_{p}, A \supset$ $B, \Gamma \vdash C$. The construction of the winning strategy for $A \supset B, \Gamma \vdash C$ is illustrated in the following picture that refers to the state transition diagram, presented above.

$$
\text { attack on } C_{p}, C_{p}, A \supset B, \Gamma \vdash A \underbrace{\alpha}_{\tau_{1}^{-}} A \supset B, \Gamma \vdash C
$$

From now on we use the term I-dialogues to denote the dialogues that have been described in this section.

\section{Hypersequent calculi for intermediate logics}

Intermediate logics (when identified with the set of its valid formulas) include intuitionistic logic and are included in classical logic. To introduce communicating parallel dialogues that are adequate for some well known intermediate logics we have to switch from sequent to hypersequent calculi.

Hypersequent calculi arise by generalizing standard sequent calculi to refer to whole contexts of sequents instead of single sequents. In our context, a hypersequent is defined as a finite, non-empty multiset of $\mathbf{L} \mathbf{I}^{\prime}$-sequents, called components; written in form

$$
\Gamma_{1} \longrightarrow C_{1}|\ldots| \Gamma_{n} \longrightarrow C_{n} \text {. }
$$

The symbol "|" is intended to denote disjunction at the meta-level.

Like ordinary sequent calculi, hypersequent calculi consist in axioms as well as logical and structural rules. The latter are divided into internal and external rules. The internal structural rules deal with formulas within components, while the external ones manipulate whole components of a hypersequent. The standard external structural rules are external weakening and external contraction:

$$
\frac{\mathscr{H}}{\Pi \longrightarrow C \mid \mathcal{H}}(E W) \quad \frac{\Pi \longrightarrow C|\Pi \longrightarrow C| \mathcal{H}}{\Pi \longrightarrow C \mid \mathcal{H}}(E C)
$$

We can disregard $(E W)$ by taking as axioms all hypersequent that contain an $\mathbf{L} \mathbf{I}^{\prime}$-axiom as component. 
The logical rules of the hypersequent $\mathbf{H L I}{ }^{\prime}$ for intuitionistic logic, are essentially the same as in $\mathbf{L I}^{\prime}$. The only difference is the presence of a side hypersequent $\mathcal{H}$, representing a (possibly empty) hypersequent. For instance, the hypersequent version of the $\mathbf{L I}$-rule $(\supset, l)$ is

$$
\frac{A \supset B, \Pi \longrightarrow A|\mathcal{H} \quad B, A \supset B, \Pi \longrightarrow C| \mathscr{H}}{A \supset B, \Pi \longrightarrow C \mid \mathscr{H}}(\supset, l)
$$

The hypersequent framework allows one to define analytic calculi for several important intermediate logics. These include Gödel-Dummett logic $\mathbf{G}_{\infty}$ (also called $\mathbf{L C}$ ) [6, 11], finite-valued Gödel logics $\mathbf{G}_{n}$ [11], and the logic $\mathbf{L} \mathbf{Q}$ of weak excluded middle [12], also called Jankov logic in reference to [12]. Adequate calculi are obtained by adding just one structural rule, respectively, to the basic hypersequent calculus $\mathbf{H L I}^{\prime}$, defined above.

- The hypersequent calculus $\mathbf{H L C} \mathbf{C}^{\prime}$ for $\mathbf{G}_{\infty}$ is obtained from $\mathbf{H L I} \mathbf{I}^{\prime}$ by adding the following rule, a version of which has already been defined in [3]:

$$
\frac{\Pi_{1}, \Pi_{2} \longrightarrow C_{1}\left|\mathcal{H} \quad \Pi_{1}, \Pi_{2} \longrightarrow C_{2}\right| \mathcal{H}}{\Pi_{1} \longrightarrow C_{1}\left|\Pi_{2} \longrightarrow C_{2}\right| \mathcal{H}}(\mathrm{com})
$$

- The hypersequent calculi $\mathbf{H G}_{k+1}^{\prime}$ for $\mathbf{G}_{k+1}$, for all $k \geq 1$, are obtained by adding to HLI' the following rules, respectively, which (essentially) were defined in [4]:

$$
\frac{\mathscr{H}\left|\Gamma_{1}, \Gamma_{2} \longrightarrow A_{1} \quad \mathcal{H}\right| \Gamma_{2}, \Gamma_{3} \longrightarrow A_{2} \quad \ldots \quad \mathcal{H} \mid \Gamma_{k}, \Gamma_{k+1} \longrightarrow A_{k}}{\mathcal{H}\left|\Gamma_{1} \longrightarrow A_{1}\right| \ldots\left|\Gamma_{k} \longrightarrow A_{k}\right| \Gamma_{k+1} \longrightarrow \perp}\left(G_{k+1}\right)
$$

Note that $\mathbf{G}_{2}$ is nothing but classical logic $\mathbf{C l}$.

- The hypersequent calculus $\mathbf{H L Q}^{\prime}$ - a variant of which was defined in [5] — is obtained from $\mathbf{H L I} \mathbf{I}^{\prime}$ by adding the following rule:

$$
\frac{\mathscr{H} \mid \Gamma, \Pi \longrightarrow \perp}{\mathscr{H}|\Gamma \longrightarrow \perp| \Pi \longrightarrow \perp}(l q)
$$

Theorem 3. $\mathbf{H L C}^{\prime}, \mathbf{H G}_{n}^{\prime}$, and $\mathbf{H L Q} \mathbf{Q}^{\prime}$ are sound and complete for the logics $\mathbf{G}_{\infty}, \mathbf{G}_{n}$, and $\mathbf{L Q}$, respectively.

Proof. Follows essentially from the soundness and cut-free completeness of the original calculi proved in [3], [4], and [5], respectively.

\section{Parallel dialogue games}

To extend the close correspondence between strongly analytic sequent proofs and winning strategies for Lorenzen style dialogues to the hypersequent level we ask the following: what happens to the winning powers of $\mathbf{P}$ if we consider games where dialogues may proceed in parallel? Of course, this question can only be answered once we have defined more precisely what we mean by 'parallel dialogue games'. Many options are open for exploration. Here, we investigate parallel versions of I-dialogue games, that share the following features: 
1. The logical and structural rules of I-games remain unchanged. Indeed, ordinary I-game dialogues appear as sub-case of the more general parallel framework.

2. The proponent $\mathbf{P}$ may initiate additional I-dialogues by 'cloning' the dialogue sequent of one of the parallel I-dialogues, in which it is her turn to move.

3. To win a set of parallel dialogues the proponent $\mathbf{P}$ has to win at least one of the component dialogues.

These items reflect basic decisions concerning 'parallelization'. In particular, it should be clear that we want to separate the level of individual dialogue moves strictly from the initiation of new dialogues and the interaction between dialogues. Moreover, we like to consider $\mathbf{P}$ as the (sole) 'scheduler' of parallel dialogues. (These features should be contrasted with alternative concepts of dialogue games, like the ones in $[1,2]$.)

Before exploring rules for the synchronization of parallel dialogues, we will investigate parallel I-dialogues as specified by conditions 1-3, alone. We will see that this results in a game that does not change the winning powers of $\mathbf{P}$ over the (single) I-dialogue game.

Notation. A parallel $\mathbf{I}$-dialogue ( $P-\mathbf{I}$-dialogue $)$ is a sequence of nodes connected by moves. Each node $v$ is labelled by a global state $\Sigma(v)$. A global state is a nonempty finite set $\left\{\Pi_{1} \vdash_{11} C_{1}, \ldots, \Pi_{n} \vdash_{n n} C_{n}\right\}$ of indexed $\mathbf{I}$-dialogue sequents. Each index $\imath k$ uniquely names one of the $n$ elements, called component dialogue sequents or simply components, of the global state. In each of the components it is either P's or O's turn to move. We will speak of a $\mathbf{P}$-component or an $\mathbf{O}$-component, accordingly. We distinguish internal and external moves.

Internal moves combine single I-dialogue moves for some (possibly also none or all) of the components of the current global state. An internal move from global state $\left\{\Pi_{1} \vdash_{11} C_{1}, \ldots, \Pi_{n} \vdash_{1 n} C_{n}\right\}$ to global state $\left\{\Pi_{1}^{\prime} \vdash_{11} C_{1}^{\prime}, \ldots, \Pi_{n}^{\prime} \vdash_{1 n} C_{n}^{\prime}\right\}$ consists in a set of indexed $\mathbf{I}$-dialogue moves $\left\{1 i_{1}: \operatorname{move}_{1}, \ldots, i_{m}:\right.$ move $\left._{m}\right\}$, such that the indices $\imath i_{j}, 1 \leq j \leq m$, are pairwise distinct elements of $\{11, \ldots, \imath n\} . \Pi_{k}^{\prime} \vdash_{\imath k} C_{k}^{\prime}$ denotes the component corresponding to the result of move ${ }_{k}$ applied to the component indexed by $\imath k$ if $k \in\left\{i_{1}, \ldots, i_{m}\right\}$; otherwise $\Pi_{k}=\Pi_{k}^{\prime}$ and $C_{k}=C_{k}^{\prime}$.

External moves, in contrast to internal moves, may add or remove components of a global state, but do not change the local status $(\mathbf{P}$ or $\mathbf{O})$ of existing components.

For now, we define only one external move, called fork.

fork is a move by $\mathbf{P}$ and consists in duplicating one of the $\mathbf{P}$-components of the current global state and assigning a new unique index to the added component.

Clearly, fork corresponds to item 2 in the above list of basic features of our parallel dialogue games. We call the new index generated by fork a child of the original index of the duplicated component.

The central condition in the definition of a $P$-I-dialogue is the following:

- each sequence of I-dialogue moves, that arises by picking at most one element $i i$ : move $_{i}$ from each of the consecutive internal moves, such that for all $1 \leq i<n$ either $1[i+1]=\imath i$ or $\mathbf{\imath}[i+1]$ is child of $\imath i$, forms an $\mathbf{I}$-dialogue.

The initial global state $\Sigma(v)$ - that is the state labelling the root node $v$ of a $P$-Idialogue - consists only of $\mathbf{O}$-components. We speak of a $P$-I-dialogue for $\Sigma(v)$. 
Example 1. We exhibit a $P$-I-dialogue for $\neg a \vee a$, for some atom $a$. Remember that $\neg a$ abbreviates $a \supset \perp$ :

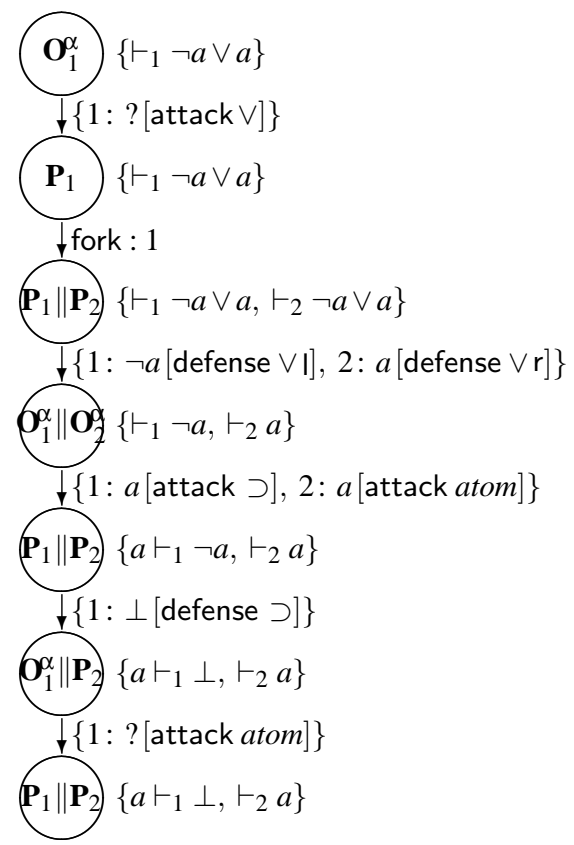

Alternative $P$-I-dialogues for $\neg a \vee a$ are possible; but it is easy to check that none of these dialogues lead to a state where player $\mathbf{P}$ is winning. However, we will see below that a special synchronization rule, which is adequate for classical logic, allows to extend this dialogue to a winning strategy for $\neg a \vee a$.

The parallel version of the dialogue game may be viewed as a finite collection of state transition systems that are coordinated by referring to a global, discrete flow of time. At each time step some (possibly also none or all) of the component dialogues advance by one move. In a fork-move the component dialogues remain in their individual current states but a new dialogue, that copies the state of one of the old ones, is created.

Observe that our definition of a $P$-I-dialogue game allows for considerable flexibility in 'implementing' the involved parallelism. We may, for example, require that all component dialogues have to advance at each time step; or, alternatively, that at most $k$ dialogues may advance simultaneously (even if there are more than $k$ components.) The latter option might, e.g., be understood as modeling a dialogue game where $\mathbf{P}$ and $\mathbf{O}$, are not single persons, but rather consist of teams of $k$ players each, and where each component dialogue is conducted by a different pair of opposite players. If, instead, we stick with a single proponent and a single opponent (i.e., $k=1$ ) it seems natural to 'sequentialize' by dove-tailing the components of parallel moves. This motivates the following definition:

- A P-I-dialogue is called sequentialized if every internal move is a singleton set.

In the proof of Theorem 1 it was essential that full cycles of moves in a winning strategy — from a $\mathbf{P}$-state to an $\mathbf{O}$-state and back again to a $\mathbf{P}$-state with an immediately 
responding move of $\mathbf{O}$ - correspond to a single inference step in $\mathbf{L I} \mathbf{I}^{\prime}$. However, even in sequentialized $P$-I-dialogues such cycles may be interrupted by internal moves referring to other components or by external moves. We therefore define a $P$-I-dialogue to be normal if the following condition holds. Every internal move that contains a $\mathbf{P}$-move indexed with $1 k$

- is immediately followed by another internal move with a $1 k$-indexed element $(\mathbf{O}$ reply),

- or, else, is the last move in the component dialogue referred to by $l k$.

Remark 2. In combination with structural rule $E$ (see Section 2), the conditions for normality can be understood as the stipulation that the proponent of a parallel dialogue game is the sole 'scheduler'. In other words - although $\mathbf{P}$ has no control over choices of $\mathbf{O}$ as long as they are immediate replies to her own previous move $-\mathbf{P}$ always determines at which dialogue component the game is to be continued.

Theorem 4. Every finite P-I-dialogue $\delta$ for $\Sigma$ can be translated into a sequentialized normal $P$-I-dialogue for $\Sigma$ ending in the same global state as $\delta$.

Proof. Sequentialization is easily achieved by replacing every internal move

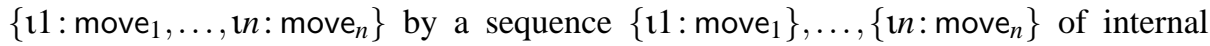
moves. (Observe that, by the definition of an internal move, the indices $t i$ are pairwise different and therefore refer to different components of a global state.)

To obtain a normal dialogue, assume that $\delta$ is already sequentialized. Unless $\delta$ is already normal, it contains a subsequence of at least three

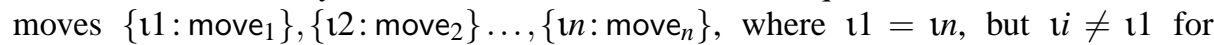
all $2 \leq i<n$, and where move $_{n}$ is an I-dialogue move by $\mathbf{O}$, that directly reacts to move $_{1}$ by $\mathbf{P}$. Clearly, reordering the sequence of moves into $\left\{\mathfrak{l} 1:\right.$ move $\left._{1}\right\},\left\{\mathfrak{l n}: \operatorname{move}_{n}\right\},\left\{12:\right.$ move $\left._{2}\right\}, \ldots,\left\{\mathfrak{l}[n-1]:\right.$ move $\left._{n-1}\right\}$ results in the same final global state. Note that - disregarding proper notation - the moves $\left\{12: \operatorname{move}_{2}\right\}$ to $\left\{\mathfrak{l}[n-1]:\right.$ move $\left._{n-1}\right\}$ may actually also be external moves without affecting the result. The claim follows by repeating this rearrangement of moves as often as possible.

Note [Important]. For the rest of the paper we will consider all parallel dialogues to be sequentialized and normal. Sequentialization implies that, just like for I-dialogues, we can speak of $\mathbf{P}$-moves and $\mathbf{O}$-moves of $\boldsymbol{P}$-I-dialogues. (fork also is a $\mathbf{P}$-move.) Since the set parentheses are redundant in denoting moves of sequentialized dialogues, we will omit them from now on.

A $P$-I-dialogue tree $\tau$ for $\Sigma$ is a rooted, directed tree with global states as nodes and edges labelled by (internal or external) moves such that each branch of $\tau$ is a $P$-Idialogue for $\Sigma$.

A finite $P$-I-dialogue tree is called a winning strategy if the following condition is satisfied for every node $v$ :

(p) either $v$ has a single successor node, the edge to which is labelled by a $\mathbf{P}$-move,

(o) or for each $\mathbf{O}$-move that is a permissible continuation of the dialogue at global state $\Sigma(v)$ there is an edge leaving $v$ that is labelled by this move,

(w) or $v$ is a leaf node and at least one of the components of $\Sigma(v)$ fulfills the winning conditions (for $\mathbf{P}$ ). 
Nodes satisfying (p) are called P-nodes; and nodes satisfying (o) are called $\mathbf{O}$-nodes. Observe that, by normality, $\mathbf{P}$-moves and $\mathbf{O}$-moves strictly alternate in each branch, except for the initial segment (consisting of more than one consecutive O-nodes, in general) and external moves (which, in general, result in consecutive $\mathbf{P}$-nodes).

Theorem 5. Every winning strategy $\tau$ for sequentialized normal P-I-dialogues with initial global state $\{\Gamma \vdash C\}$ can be transformed into an $\mathbf{H L I}^{\prime}$-proof of $\Gamma \longrightarrow C$.

Proof. We show by induction on the depth of $\tau$ that for every $\mathbf{P}$-node of $\tau$ labelled with global state $\Sigma$, there is an $\mathbf{H L I} \mathbf{I}^{\prime}$-proof of the corresponding hypersequent $[\Sigma]$. Since the branches of $\tau$ are normal and sequential dialogues, edges of $\tau$ that correspond to internal moves are translated into corresponding inference steps using logical rules of $\mathbf{H L I} \mathbf{I}^{\prime}$, exactly as in the proof of Theorem 1. Moreover, if the winning condition is fulfilled for one of the component dialogues, then the global state clearly corresponds to an axiom of $\mathbf{H L I}$.

It remains to show that also fork translates into external contraction $(E C)$ : Suppose ( $\vee$ - $\longrightarrow$ ( $v$ is an edge of $\tau$ corresponding to a last fork-move of a branch of $\tau$. Then the global state at $\Sigma\left(v^{\prime}\right)$ is like $\Sigma(v)$ except for an additional dialogue sequent $\Gamma \vdash_{1 i} A$, where the index $i i$ is not yet used at $v$, but where, for some $1 j, \Gamma \vdash j A$ is an element of $\Sigma(v)$. Clearly, the required $\mathbf{H L I} \mathbf{I}^{\prime}$-proof of $[\Sigma(v)]$ is obtained from $\pi_{v^{\prime}}$ by adding an appropriate application of $(E C)$ as the last inference.

Again, we call cut-free proofs without applications of (internal or external) weakening or internal contraction strongly analytic.

Theorem 6. Every strongly analytic $\mathbf{H L I}^{\prime}$-proof $\pi$ of the hypersequent $\Gamma \longrightarrow C$ can be transformed into a winning strategy $\tau$ for $P$-I-dialogues for $\{\Gamma \longrightarrow C\}$.

Proof. Since $\pi$ is strongly analytic, there are no applications of internal structural rules. The logical rules of $\mathbf{H L I} \mathbf{I}^{\prime}$ translate into full $\mathbf{P}-\mathbf{O}-\mathbf{P}$-cycles of internal moves, exactly as in the proof of Theorem 2. It remains to show that external weakenings correspond to fork-moves for $P$-I-dialogues. It suffices to consider a sub-proof of $\pi$ ending with the inference

$$
\frac{\Pi \longrightarrow D|\Pi \longrightarrow D| \mathcal{H}}{\Pi \longrightarrow D \mid \mathcal{H}}(E C)
$$

By induction hypothesis there exists a winning strategy $\tau^{\prime}$ for $\left\{\Pi \vdash_{11} D\right\} \cup\left\{\Pi \vdash_{12} D\right\} \cup$ $\langle\mathcal{H}\rangle$, which has to be extended to one for $\left\{\Pi \vdash_{11} D\right\} \cup\langle\mathcal{H}\rangle$, where $\langle\mathcal{H}\rangle$ denotes the set of dialogue sequents corresponding to the components of $\mathcal{H}$. This is easily achieved by inserting a new edge corresponding to an appropriate instance of the fork-move immediately after the initial (internal $\mathbf{O}$-)move of $\tau^{\prime}$.

\section{Synchronizing dialogues}

Synchronization between I-dialogues is formalized as merging of two or more dialogues into one according to the following general principle: $\mathbf{P}$ selects (for merging) some P-components from the global state. The picked components are then merged into a 
new dialogue in some straightforward way. For some synchronization rules, there are different possible ways to merge the components picked by $\mathbf{P}$. In those cases $\mathbf{O}$ may choose one of them.

In [10] the following (two-part) synchronization rule for Gödel-Dummett logic $\mathbf{G}_{\infty}$ was already discussed briefly:

Ic- P-part: $\mathbf{P}$ picks two (indices of) $\mathbf{P}$-components $\Pi_{1} \vdash_{11} C_{1}$ and $\Pi_{2} \vdash_{12} C_{2}$ from the current global state and thus indicates that $\Pi_{1} \cup \Pi_{2}$ will be the granted formulas of the resulting merged dialogue sequent.

Ic- O-part: In response to this external $\mathbf{P}$-move, $\mathbf{O}$ chooses either $C_{1}$ or $C_{2}$ as the active formula of the merged component, which is indexed by 11 or 12 , correspondingly.

Not only infinite valued Gödel logic can be characterized by an appropriate parallel dialogue game, but also each of the $n$-valued Gödel logics $\mathbf{G}_{n}$. Here is the appropriate synchronization rule, parameterized by $n$, where $n \geq 2$ :

$\mathrm{g}_{n}$ - P-part: $\mathbf{P}$ picks $n-1 \mathbf{P}$-components $\Pi_{1} \vdash_{11} C_{1}, \ldots \Pi_{n-1} \vdash_{1[n-1]} C_{n-1}$, and a $\mathbf{P}$ component of form $\Pi_{n} \vdash_{1 n} \perp$ from the current global state for merging.

$g_{n}$ - O-part: $\mathbf{O}$ chooses one of the components $\Pi_{1} \cup \Pi_{2} \vdash_{11} C_{1}, \Pi_{2} \cup \Pi_{3} \vdash_{12} C_{2}, \ldots$ or $\Pi_{n-1} \cup \Pi_{n} \vdash_{\mathfrak{1}[n-1]} C_{n-1}$ as the merged component, that replaces the components picked by $\mathbf{P}$.

Note that for the case of two truth values, i.e., for classical logic, no proper choice is left for $\mathbf{O}$; hence $g_{2}$ can be stated simpler as follows:

$\mathrm{cl}\left(=\mathrm{g}_{2}\right)$ : If the global state contains a $\mathbf{P}$-component of form $\Pi \vdash_{1} \perp$ then $\mathbf{P}$ may remove this component and add $\Pi$ to the granted formulas of another $\mathbf{P}$-component of the global state.

In other words, if $\mathbf{P}$ detects that in one of the components she faces the task to defend falsum, then she may cancel the corresponding I-dialogue while transferring its currently granted formulas to another $\mathbf{P}$-component of her choice.

Example 2. Rule $\mathrm{cl}$ allows $\mathbf{P}$ to continue the parallel dialogue for $\neg a \vee a$ in Example 1 as follows:

$$
\begin{aligned}
& \mathbf{P}_{1} \| \mathbf{P}_{2}\left\{a \vdash_{1} \perp, \vdash_{2} a\right\} \\
& \text { lcl : 1,2 } \\
& \text { (P) }\left\{a \vdash_{1} a\right\} \\
& \mathbf{P} \text { wins! }
\end{aligned}
$$

Replacing $\mathrm{cl}$ by the following subtle variant, allows to characterize Jankov logic LQ, which allows $\mathbf{P}$ to win every dialogue for a formula of form $\neg A \vee \neg \neg A$ :

lq: If the global state contains a $\mathbf{P}$-component of form $\Pi \vdash_{1} \perp$ then $\mathbf{P}$ may remove this component and add $\Pi$ to the granted formulas of another $\mathbf{P}$-component of the global state, which also has $\perp$ as active formula.

We summarize the above synchronization rules and provide names to the resulting systems of parallel dialogue games in the following table. 


\section{Parallel dialogue games extending $P$-I-games:}

(All dialogue sequents exhibited in the table are $\mathbf{P}$-components)

\begin{tabular}{|c|c|l|}
\hline System & Rule & Synchronization (external merging move) \\
\hline \hline$P$-G & Ic & $\begin{array}{l}\mathbf{P} \text { wants to merge } \Pi_{1} \vdash_{11} C_{1} \text { and } \Pi_{2} \vdash_{12} C_{2} \\
\mathbf{O} \text { chooses either } \Pi_{1} \cup \Pi_{2} \vdash_{11} C_{1} \text { or } \Pi_{1} \cup \Pi_{2} \vdash_{12} C_{2}\end{array}$ \\
\hline$P$-G & $\mathrm{g}_{n}$ & $\begin{array}{l}\mathbf{P} \text { wants to merge } \Pi_{1} \vdash_{11} C_{1} \text {, and } \ldots \Pi_{n-1} \vdash_{1[n-1]} C_{n-1}, \text { and } \Pi_{n} \vdash_{1 n} \\
\mathbf{O} \text { chooses either } \Pi_{1} \cup \Pi_{2} \vdash_{11} C_{1}, \Pi_{2} \cup \Pi_{3} \vdash_{12} C_{2}, \ldots \text { or } \Pi_{n-1} \cup \Pi_{n} \vdash_{1[n-1]} C_{n-1}\end{array}$ \\
\hline$P$-Cl & $\mathrm{cl}=\mathrm{g}_{2}$ & $\mathbf{P}$ merges $\Pi \vdash_{11} \perp$ and $\Gamma \vdash_{12} C$ into $\Pi \cup \Gamma \vdash_{12} C$ \\
\hline$P$-LQ & lq & $\mathbf{P}$ merges $\Pi \vdash_{11} \perp$ and $\Gamma \vdash_{12} \perp$ into $\Pi \cup \Gamma \vdash_{12} \perp$ \\
\hline
\end{tabular}

Let us call parallel dialogues that are defined exactly as $P$-I-dialogues, except for including one of the rules lc, $\mathrm{g}_{n}$, or lq, $P-\mathbf{G}-, P-\mathbf{G}_{n^{-}}$, and $P$-LQ-dialogues, respectively.

Theorem 7. Every winning strategy $\tau$ for sequentialized normal $P-\mathbf{G}-\left(P-\mathbf{G}_{n^{-}}\right.$, or $P$ LQ-) dialogues with initial global state $\left\{\Gamma \vdash_{1} A\right\}$ can be transformed into an $\mathbf{H L C}$ $\left(\mathbf{H G}_{n}^{\prime}{ }^{-}\right.$, or $\mathbf{H L} \mathbf{Q}^{\prime}$-) proof $\pi$ of the corresponding hypersequent $\Gamma \longrightarrow A$, and vice versa.

Proof. Given the proofs of Theorems 5 and 6, it remains to show that the synchronization rules lc, $\mathrm{g}_{n}$, and lq correspond to the hypersequent rules (com), $\left(\mathrm{g}_{n}\right)$, and (lq), respectively. We present the case for Ic/(com); the other cases are similar or simpler.

$(\Rightarrow)$. Suppose $\vee \longrightarrow$ iv is an edge of $\tau$ which corresponds to an instance of Ic. The relevant part of $\tau$ looks as follows. We use $F_{p}$ to denote $A$ if $F$ is of form $A \supset B$; otherwise $F_{p}$ is empty:

$$
\text { Ic [O-response]: } 11 \text { (1) } \Sigma\left(v_{0}\right),\left\{C_{p}, \Pi_{1} \vdash_{11} C, D_{p}, \Pi_{2} \vdash 2 D\right\} \subseteq \Sigma\left(v_{0}\right)
$$

where $\Sigma\left(\mathrm{v}^{\prime}\right)=\Sigma(\mathrm{v})-\left\{C_{p}, \Pi_{1} \vdash_{11} C, D_{p}, \Pi_{2} \vdash_{12} D\right\} \cup\left\{C_{p}, \Pi_{1}, D_{p}, \Pi_{2} \vdash_{11} C\right\}$ and $\Sigma\left(v^{\prime \prime}\right)=\Sigma(v)-\left\{C_{p}, \Pi_{1} \vdash_{11} C, D_{p}, \Pi_{2} \vdash_{12} D\right\} \cup\left\{C_{p}, \Pi_{1}, D_{p}, \Pi_{2} \vdash_{12} D\right\}$. By induction hypothesis there exist $\mathbf{H L C} \mathbf{C}^{\prime}$-proofs $\pi_{v^{\prime}}$ and $\pi_{v^{\prime \prime}}$ of the corresponding hypersequents $\left[\Sigma\left(v^{\prime}\right)\right]$ and $\left[\Sigma\left(v^{\prime \prime}\right)\right]$, respectively. Clearly, $\pi_{v^{\prime}}$ and $\pi_{v^{\prime \prime}}$ can be joined by an application of (com) to obtain the required proof of $\left[\Sigma\left(v_{0}\right)\right]$.

$(\Leftarrow)$ Suppose $\pi$ contains a subproof that ends in an application of the communication rule. (To make the proof more transparent we disregard side hypersequents.)

$$
\frac{\frac{\vdots}{\Pi_{1}, \Pi_{2} \longrightarrow C} \quad \frac{\vdots}{\Pi_{1}, \Pi_{2} \longrightarrow D}}{\Pi_{1} \longrightarrow C \mid \Pi_{2} \longrightarrow D}(\mathrm{com})
$$

By induction hypothesis there exist winning strategies $\tau_{1}$ and $\tau_{2}$ for $\left\{\Pi_{1}, \Pi_{2} \vdash_{11} C\right\}$ and $\left\{\Pi_{1}, \Pi_{2} \vdash_{12} D\right\}$, respectively, that are of following form:
(О) $\left\{\Pi_{1}, \Pi_{2} \vdash_{\mathrm{l} 1} C\right\}$
1: attack on $C$
$\mu_{1}: \bigodot_{\tau_{1}^{\prime}}\left\{C_{p}, \Pi_{1}, \Pi_{2} \vdash 11 C\right\}$
(О) $\left\{\Pi_{1}, \Pi_{2} \vdash_{12} D\right\}$
2: attack on $D$
$\mu_{2}: \bigodot_{\tau_{2}^{\prime}}^{\mathbf{P}}\left\{D_{p}, \Pi_{1}, \Pi_{2} \vdash_{12} D\right\}$ 
A winning strategy for $\left\{\Pi_{1} \vdash_{11} C, \Pi_{2} \vdash_{12} D\right\}$ is obtained by attaching to

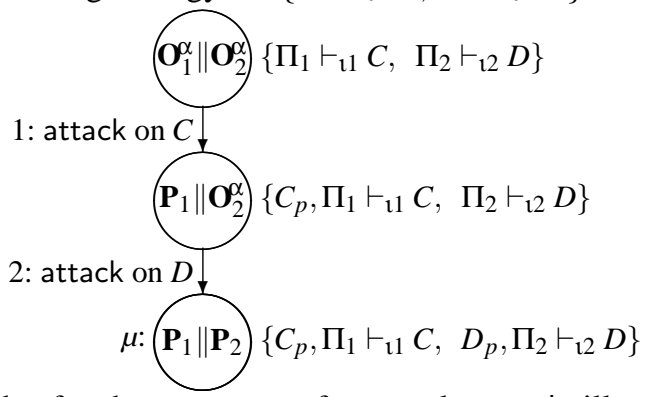

the nodes for the two parts of external move Ic illustrated in case $(\Rightarrow)$, above, where node $\mu$ is identified with node $v_{0}$. Finally, the sub-strategies $\tau_{1}^{\prime}$ and $\tau_{2}^{\prime}$ are attached by identifying node $v^{\prime}$ with node $\mu_{1}$ and node $v^{\prime \prime}$ with node $\mu_{2}$.

Acknowledgement. I thank the referees and Agata Ciabattoni for useful comments.

\section{References}

1. S. Abramsky, R. Jagadeesan: Games and Full Completeness for Multiplicative Linear Logic. J. Symbolic Logic, 59(2) (1994), 543-574.

2. A. Blass: A Game Semantics for Linear Logic. Annals of Pure and Applied Logic, 56(1992), $183-220$

3. A. Avron. Hypersequents, logical consequence and intermediate logics for concurrency. Annals of Mathematics and Artificial Intelligence, 4(1991), 225-248.

4. A. Ciabattoni, M. Ferrari. Hypersequent calculi for some intermediate logics with bounded Kripke models. Journal of Logic and Computation, 2(11), pp. 283-294, 2001.

5. A. Ciabattoni, D.M. Gabbay, N. Olivetti. Cut-free proof systems for logics of weak excluded middle. Soft Computing, 2(4), pp 147-156, 1998.

6. M. Dummett. A propositional calculus with denumerable matrix. J. Symbolic Logic, 24(1959), 97-106.

7. J.M. Dunn, R.K. Meyer. Algebraic completeness results for Dummett's $L C$ and its extensions. Z. Math. Logik Grundlagen Math., 17 (1971), 225-230.

8. W. Felscher: Dialogues, Strategies, and Intuitionistic Provability. Annals of Pure and Applied Logic, 28(1985), 217-254.

9. W. Felscher: Dialogues as Foundation for Intuitionistic Logic. In: D. Gabbay and F. Günther (eds.), Handbook of Philosophical Logic, III, Reidel, 1986, 341-372.

10. C.G. Fermüller, A. Ciabattoni. From Intuitionistic Logic to Gödel-Dummett Logic via Parallel Dialogue Games. 33rd Intl. Symp. on Multiple-Valued Logic, Tokyo May 2003, IEEE Press, 188-193.

11. K. Gödel: Zum intuitionistischen Aussagenkalkül. Anz. Akad. Wiss. Wien, 69(1932), 65-66.

12. V. Jankov. The calculus of the weak "law of excluded middle". Mathematics of the USSR $\mathbf{8}$, pp. 648-658, 1968.

13. E.C.W. Krabbe: Formal Systems of Dialogue Rules. Synthese, 63(1985), 295-328.

14. E.C.W. Krabbe: Dialogue Sequents and Quick Proofs of Completeness. In: J.Ph. Hoepelman (ed.), Representation and Reasoning. Max Niemeyer Verlag, 1988, 135-140.

15. P. Lorenzen: Logik und Agon. In: Atti Congr. Internat. di Filosofia, Vol. 4 (Sansoni, Firenze, 1960), 187-194.

16. S. Rahman: Über Dialoge, Protologische Kategorien und andere Seltenheiten. Europäische Hochschulschriften, Peter Lang, 1993. 